\title{
Performance Analysis of a Surveillance System to Detect and Track Vehicles using Haar Cascaded Classifiers and Optical Flow method
}

\author{
Kazy Noor E Alam Siddiquee \\ Department of Computer Science and Engineering \\ University of Science and Technology Chittagong \\ Foy's Lake, Chittagong, Bangladesh \\ Dhiman Sarma \\ Department of Computer Science and Engineering \\ Rangamati Science and Technology University \\ Rangamati
}

\section{Avijit Nandi}

Department of Computer Science and Engineering University of Science and Technology Chittagong

Foy's Lake, Chittagong, Bangladesh

\section{Sharmin Akhter}

Department of Computer Science and Engineering University of Science and Technology Chittagong

Foy's Lake, Chittagong, Bangladesh

\author{
Sohrab Hossain \\ Department of Computer Science and Engineering \\ University of Science and Technology Chittagong \\ Foy's Lake, Chittagong, Bangladesh
}

\author{
Karl Andersson \\ Pervasive and Mobile Computing Laboratory \\ Luleå University of Technology \\ SE-931 87 Skellefteå, Sweden \\ Mohammad Shahadat Hossain \\ Department of Computer Science and Engineering \\ University of Chittagong \\ Chittagong
}

\begin{abstract}
This paper presents the real time vehicle detection and tracking system, based on data, collected from a single camera. In this system, vehicles are detected by using Haar Feature-based Cascaded Classifier on static images, extracted from the video file. The advantage of this classifier is that, it uses floating numbers in computations and hence, $20 \%$ more accuracy can be achieved in comparison to other classifiers and features of classifiers such as LBP (Local Binary Pattern). Tracking of the vehicles is carried out using Lucas-Kanade and Horn Schunk Optical Flow method because it performs better than other methods such as Morphological and Correlation Transformations. The proposed system consists of vehicle detection and tracking; and it is evaluated by using real data, collected from the route networks of Chittagong City of Bangladesh.
\end{abstract}

Keywords-Haar like Features, Cascade Classifiers, vehicle detection, vehicle tracking

\section{INTRODUCTION}

Cameras are now-a-days widely used to detect and track the vehicles in the route network systems of a country. Consequently, substantial increase in surveillance is noticed. However, the accuracy of vehicle detection and tracking is considered as crucial to make the surveillance a robust one. Therefore, the deployment of appropriate methods and technologies is essential to support vehicle detection and tracking efficiently. One of these methods is the use of Haar Feature based Cascaded Classifier to detect the vehicles in a route network.

Haar Feature-based Cascaded Classifier, used in object detection was the first real time face detector. Cascade classifiers contains list of states and each of them contains a list of weak learners. Haar like features train those cascade functions from lots of positive and negative images. It reduced complexity of feature calculations in images as RGB pixel values whereas methods used in other photogrammetric imageries were computationally expensive [1], [2].

Prime advantage of Haar like features is its calculation speed. It can use integral images and can be processed in constant time. It takes 60 microprocessor instructions for a 2 rectangle feature, approximately.

The tilted (45 degree) Haar like feature approach was proposed in [3] to increase dimensionality, allowing the improvement of object detection techniques. However, this approach was extended into a new one, known as generic rotated Haar like feature [4]. The later approach prevents the use of Haar like features (at any angle) in some cases, although it is mathematically efficient. Most Detection algorithms [1],[2],[3],[4],[5] use low resolution images to 
detect object quickly by rounding errors. Haar Like features with cascaded classifiers have shown better performance than those of two approaches. Hence, these two approaches are used rarely now -a- days.

In this paper, Haar Feature based Cascaded classifiers have been used. With this method, the detection is performed on static images collected from a video file by using single camera. While Lucas-Kanade Optical Flow method can be used to track vehicles in a route network. It performs better than other methods as it is relatively easier to implement and it has faster calculating speed than Morphological and Correlation Transformations as will be shown. Therefore, Haar Feature based Cascaded Classifier along with LucasKanade Optical Flow methods have been considered to develop the surveillance system in this paper. Figure 1 illustrates the architectural block diagram of the proposed surveillance system.

As, tracking of vehicles is another concern of this paper, both the learning algorithm using Haar cascaded classifier and the optical flow method have been chosen. Morphological features and correlation transformations could have been used. But from the point of view of two parameters (1. number of vehicles in a road at a certain moment and 2 . the frame rate (while the video is being captured)), the graphical comparison shows that the optical flow method is better than the other two. Experiments for Haar feature based on cascaded classifier and Lucas Kanade methods on benchmarks with real time data have been collected.

The paper is organized with 4 sections more. Section 2

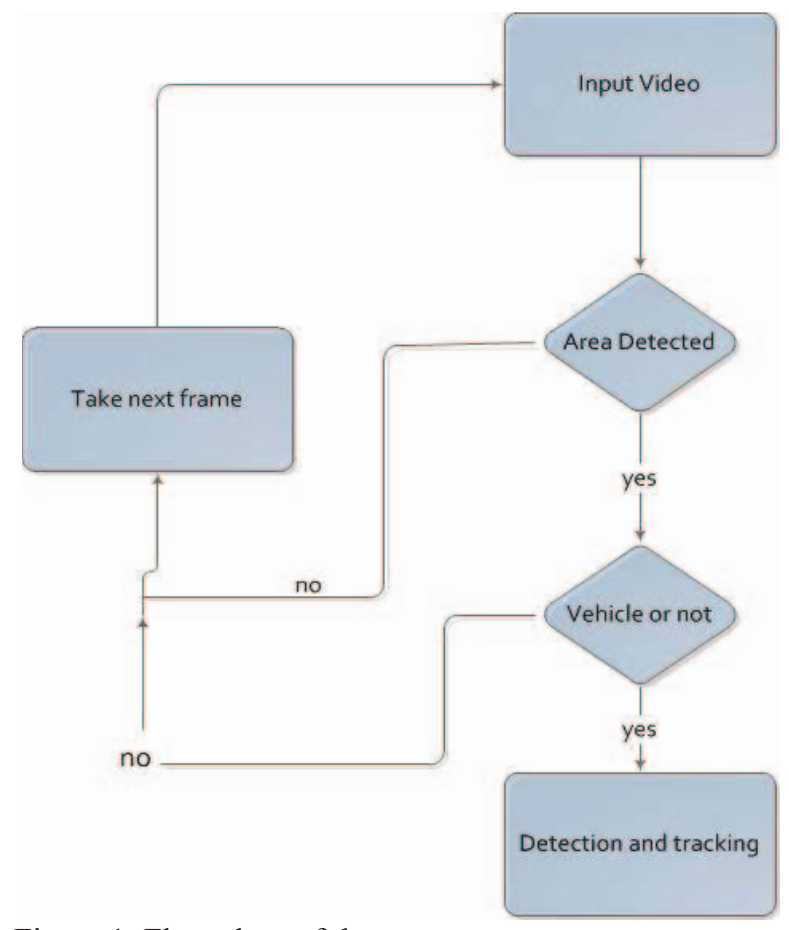

Figure 1: Flow chart of the system discusses on current research approaches used in vehicle detection and tracking while section 3 highlights on research methodologies. This section is divided into two parts, first one describes the algorithms and methods for vehicle detection and the second part describes the tracking method of vehicles.

\section{RELATED WORK}

Object detection in static images is a challenging problem in the field of computer vision. Vehicles are taken into consideration for both detection and tracking for keeping records of vehicle information and keeping road accidents under surveillance [5], [6]. Earlier, many approaches had been proposed for vehicle detection and tracking. Background subtraction method was the pioneering technique among all. Later on, detection and tracking techniques were aiming statistical methods like SVM [5]. Statistical methods were utilized to overcome shortcomings of basic background subtraction methods and emerged as reliable techniques for processing video frames containing noise, illumination changes and shadows. Real time vehicle detection and tracking techniques were brought into consideration by the cascaded classifiers of Viola and Jones algorithms first [1]. Viola Jones algorithm proposed a method for training a sequential classifier working on simple-to-evaluate Haar-like features and demonstrated its real-time performance on the face detection problem.

In paper [7], for human face detection near infrared technique was approached. OpenCV based on Haar cascade Classifier have been used. Haar cascade classifiers were also used in object detection in ultrasound images [8] and the detector was trained by AdaBoost algorithm. Detection was $96.29 \%$ successful compared to SVM classification result in paper [8]. A significant attention was grown to vehicle detection and various filter algorithms were primary focus for detection of vehicles. Kalman filter was used to detect vehicles from roadside CCTV videos in paper [9]. The detection rate was $96.39 \%$ and in challenging weather it was $94.69 \%$. Robust and reliable vehicle detection successfully got implemented using MMW radar and Mono-vision Fusion (monocular camera) in [10]. From real world data set, the experiment showed $92.36 \%$ result. Earlier to this research the log Gabor filter was optimized for improved vehicle detection in [11]. In this paper challenges of heterogeneity of vehicles (in color, size, pose etc) was reduced although Gabor filter had wide limitations on frequency responses. Haar cascade classifiers were afterwards considered to be used for vehicle detection and real time vehicle detection methods replaced statistical detection methods. Uses of Haar-SURF mixed features and gentle AdaBoost classifier for real time vehicle detection was found in [12]. The detection algorithm used cascade classifiers which was trained by Gentle AdaBoost classifier with HaarSURF mixed features. Afterwards, in 2016 the detection got improved with foreground based Haar like features and cascade classifiers [13]. The algorithm was successfully evaluated and implemented with public datasets. AdaBoost classifier with Haar feature decision stumps were used for detection of vehicles for parking at early stages [14]. A multi- 
scan detection technique with a Soft cascade classifiers design was proposed by using Adaboost (with Haar like features) and afterwards, image segmentation with real time vehicle detection had been implemented. This approach proposed an improved accuracy compared to traditional Adaboost methods. VeDAS-Vehicle Detection using active learning and Symmetry was another dimensional approach using Haar like features. VeDAS was able to detect partial rear views of vehicles [15]. The success rate was $87 \%$ on dataset of new partially visible rear view vehicles.

The Lucas Kanade Optical Flow method was developed in 1981 by Bruce D Lucas and Takeo Kanade [16]. In this paper, for detection and tracking of vehicles, Lucas-Kanade method offered by libraries in MATLAB is adopted for tracking of vehicles. This method uses flow vectors of motion objects in time in images of a video file. Instead of being time consuming, this method was adopted for calculation of apparent velocity and direction of motions in frames in terms of pixels. Independent motions of vehicles are also detected. The result of vehicle detection is compared between Haar like cascaded classifiers and LBP cascaded classifiers. Then, the result of tracking (using optical flow method) is compared with Morphological features and Correlation Transformation. A vehicle detection algorithm was proposed in paper [17] based on the morphology and wavelet transform, in the context of the traditional difference. In paper [18] a new method was adopted which was an approach that combined Brox's optical flow algorithm and background subtraction method to improve the optical flow in the traffic monitoring system.

In paper [19], authors discussed and addressed the issue of detecting vehicle/traffic data from video frames. The method was invented with an increased real time video processing proposing a unique algorithm. For vehicle recognition and tracking Parallel Optical Flow method based on Lucas-Kanade algorithm was used. The foreground optical flow detector detects the object and a binary computation is done to define rectangular regions around every detected object. To detect the moving object correctly and to remove the noise some morphological operations have been applied. Then the final counting is done by tracking the detected objects and their regions in a real time sequence.

\section{OVERVIEW OF METHODOLOGY}

In this paper, full-featured vehicle detection and tracking system is particularly designed to work on video footage. This system is mainly having two modules:-

- Video Acquisition.

- Video detection and tracking

The system is designed for real time videos where a camera is used for continuous recording of videos. A defined connected component area is taken as threshold; if the detected area is above that threshold value then it will be recognized as a vehicle and will be tracked. A distance is defined between the vehicle and the camera. When the vehicle comes within that range i.e vehicle's connected component area is maximum. The flow chart of the system is shown in Figure 1 and figure 2.

\section{a) Object Detection}

The detection of vehicles is carried out using a learning algorithm and the Learning algorithm is given below -

The algorithm forms a strong classifier with the linear combination of weak classifiers.

$$
f(x)=\operatorname{sign}\left(\sum_{i=0}^{n} a_{i} f_{i}(x)\right)
$$

There is a threshold function based on the feature $t_{i}$

$$
f_{i}(x)=\left\{\begin{array}{cc}
-g_{i} & \text { if } t_{i}<\theta_{i} \\
g_{i} & \text { otherwise }
\end{array}\right.
$$

$\theta_{i}$ and polarity of $g_{i} \in \mp 1$ are determined in training with the coefficients $a_{i}$

Input: There are set of $\mathrm{N}$ positive and negative training images $\left(x^{j}, y^{j}\right)$. If imagery $\mathrm{j}$ is a face $\mathrm{y}^{\mathrm{j}}=1$; if not, $\mathrm{y}^{\mathrm{j}}=-1$

1. Assign weight $\omega_{1}^{j}=\frac{1}{N}$ to each image $\mathrm{j}$

2. For each $\mathrm{f}_{\mathrm{i}}, \mathrm{i}=1 \ldots \ldots \mathrm{M}$

a. renormalize weights such that they sum to 1

b. apply features to each image in training set and find optimal threshold and polarity of $\theta_{i}, g_{i}$ for minimizing the weighted classification error

$$
\begin{aligned}
& \theta_{i}, g_{i}=\underset{\theta, s}{\operatorname{argmin}} \sum_{j=0}^{n} \omega_{i}^{j} \varepsilon_{i}^{j} \quad \text { where } \\
& \varepsilon_{i}^{j}=\left\{\begin{array}{l}
0 \text { if } y^{j}=f_{i}\left(x^{j}, \theta_{i}, g_{i}\right) \\
1 \quad \text { otherwise }
\end{array}\right. \\
& \text { c. assign a weight } a_{i} \text { to } f_{i} \text { which is inversely }
\end{aligned}
$$
proportional to the error rate

d. weights for next iteration (for example $\omega_{i+1}^{j}$ ) are lessened for images $\mathrm{j}$ those were classified correctly.

3. Finalize classifier to $f(x)=\operatorname{sign}\left(\sum_{i=0}^{n} a_{i} f_{i}(x)\right)$

b) Object tracking

This section describes the vehicle tracking techniques and the Lucas-Kanade Optical Flow method has been adopted for this purpose.

Lucas-Kanade Optical Flow method [16] is added to measure the accuracy of tracking and OpenCV provides all these in a single function ${ }^{1}$ for vehicle detection and tracking where optical flow library of MATLAB has been used for tracking of vehicles. This function uses two variables; the vector data is $\mathrm{V}=(\mathrm{u}, \mathrm{v})$ ( $\mathrm{v}$ means, pixels of moving object's direction and variation of pixels in time in different frames) whereas frame intensity is denoted by by $\mathrm{I}(\mathrm{x}, \mathrm{y}, \mathrm{t})$, in the following equation (Taylor Expansion) in terms of $\mathrm{t}$ along with $\mathrm{x}$ and $\mathrm{y}$ (from point $A$ with a time $t$ to point $B$ with the extended time $d t$ ).

\footnotetext{
${ }^{1}$ http://docs.opencv.org/trunk/d7/d8b/tutorial_py_lucas_kanade.html
} 
$I(x+d x, y+d y, t+d t)=I(x, y, t)+\partial I / \partial x . d x+\partial I / \partial y . d y+$ $\partial I / \partial t . d t+\cdots$

This equation can also be represented as below-

$I(x,+1)=I(x+V x, y+v y, t)$

$\mathrm{V}=(\mathrm{Vx}, \mathrm{Vy})$ shows the velocity of vector and the derivatives outcome following constraint equation for optical flow-

$$
\left(\underset{v l}{\rightarrow} \cdot \underset{v}{\partial t}+\frac{\partial I}{\partial t}=0\right)
$$

Here $\underset{v}{\rightarrow}=\left(\frac{\partial l}{\partial x}, \frac{\partial l}{\partial y}\right)$ is the gradient (in frame). Lucas-Kanade will work on the the optical flow on the $\mathrm{u}, \mathrm{v}$ point. The motion vector is characterized by variable $\theta=(a, b)$. The deviation from the $(\mathrm{u}, \mathrm{v})$ can be evaluated by using Lucas-Kanade search vector $\mathrm{V}$ by following equation:

$\vec{v}(u, v)=\operatorname{argminwith} \theta \sum_{x, y \in \theta u, v}\left[\frac{\partial l}{\partial x} \cdot a+\frac{\delta l}{\delta y} \cdot b+\frac{\partial l}{\partial t}\right] 2$

If a vehicle is tracked in a frame, it is tracked in all consecutive frames where it is visible. Thus, it avoids redundantly and repeatedly detection of a single objects multiple times.

Lucas-Kanade optical method takes arithmetic differentials of every 2 frames and used to detect optical flow of vehicles. The process of the optical flow is depending on the central value of pixels which are related to local variables.

\section{RESULT AND DISCUSSION:}

The system is designed for vehicle detection and tracking of vehicles on roads and highways in Chittagong City of Bangladesh using a single camera mounted on a moving or stationary car. The system is running in real-time $(10 \mathrm{~Hz})$ on a single CPU core. We opted for a single camera-based system since it is cheap, use a minimum energy; it is light and weight robust. It can easily by mount on a motorbike or even, forward or rear facing on a bicycle. A multiple single camera systems with inconsistent viewing direction, angles and distance ranges can also be deployed.

The system was tested on an Intel Core Duo (1.83 GHZ) CPU and 2GB RAM. Data sets were collected from image sequences of highway scenes. The system is able to track and detect most vehicles successfully. Following figures show some results of our system.

Results are shown from input image in daylight view in figure 3. Then the Blob Tracking of vehicle and PBAS are shown in figure 4 and figure 5 respectively. In low light data, input is shown in figure 6 , BLOB for tracking of vehicles at low light in figure 7. Tracking result using optical flow method is shown in figure 8, 9, 10 and 11.

The OpenCV utilizes a path to a video file and then a pointer is created. For a width of 500 pixels, images are converted to grayscale and Gaussian blurring is used to smooth images out of high frequency noises since, these noises can throw the detection algorithm off. Small changes to the lightings may trigger false-positives. OpenCV reduces this problem significantly.

Results of detection of Haar cascades are compared to performances of a feature such as LBP cascades. LBP is faster as it calculates with integers whereas Haar cascades uses floating numbers which grabs time more. But, processing of Haar is more accurate $(10-20 \%)$ than that of LBP and the result is obtained from the collected dataset in Bangladesh motorways using a single camera and given in both Table I and Table II.

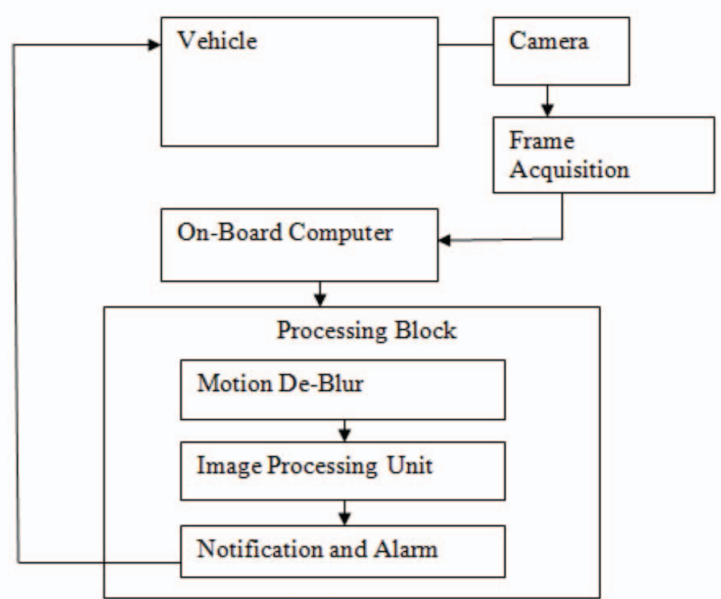

Figure 2: Basic Block Diagram for tracking of vehicles

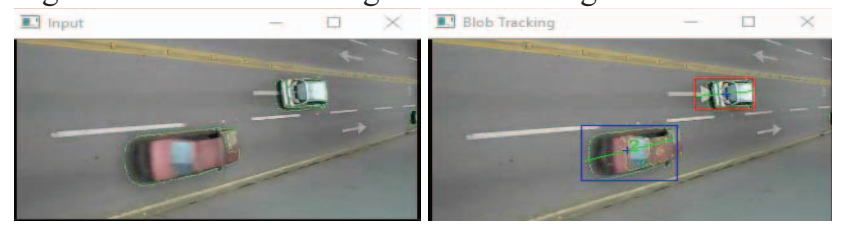

Figure 3

Figure 4

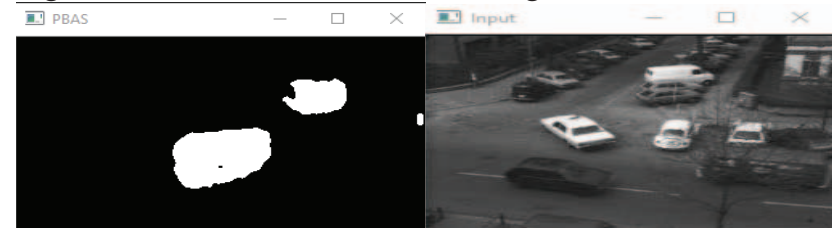

Figure 5

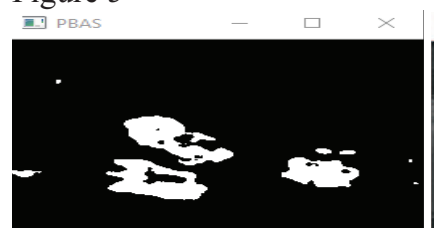

Figure 7



Figure 9
Figure 6

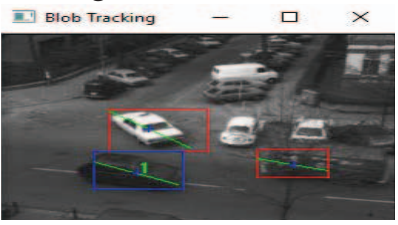

Figure 8

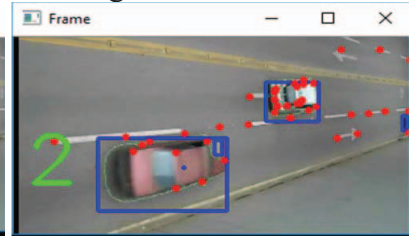

Figure 10 


\begin{tabular}{|c|c|c|}
\hline No of Vehicles & Efficiency in CT & Efficiency in MT \\
\hline 1 & 80 & 80 \\
\hline 2 & 80 & 70 \\
\hline 5 & 70 & 50 \\
\hline 10 & 60 & 43 \\
\hline 12 & 43 & 20 \\
\hline & - \\
\hline
\end{tabular}

Figure 11

Figure 3: Input image (at day time)

Figure 4: blob tracking (at day time)

Figure 5: PBAS frame (at day time)

Figure 6: Input at low light

Figure 7: PBAS frame at low light

Figure 8: BLOB for tracking of vehicles at low light

Figure 9: detection and tracking in optical flow method (LK)

Figure 10: Output of detection and tracking in LK method

Figure 11: Output of detection and tracking in LK method

TABLE I: HAAR CASCADE(TIME: 4M 2.060S)

\begin{tabular}{|c|c|c|c|}
\hline Hits & Misses & False Detects & Multi-hit \\
\hline 3635 & 55 & 63 & 5 \\
\hline
\end{tabular}

TABLE II: LBP CASCADE(TIME: 1M 12.511s)

\begin{tabular}{|c|c|c|c|}
\hline Hits & Misses & False Detects & Multi-hit \\
\hline 3569 & 106 & 77 & 3 \\
\hline
\end{tabular}

Training of Haar cascades is 3 times slower than that of LBP cascades. Still, Haar cascade is $1-2 \%$ accurate more on detection of vehicles and the detection rate is $95 \%+$ according to given dataset given in above tables. Here, multi-hit resembles to number of detection of the same vehicle.

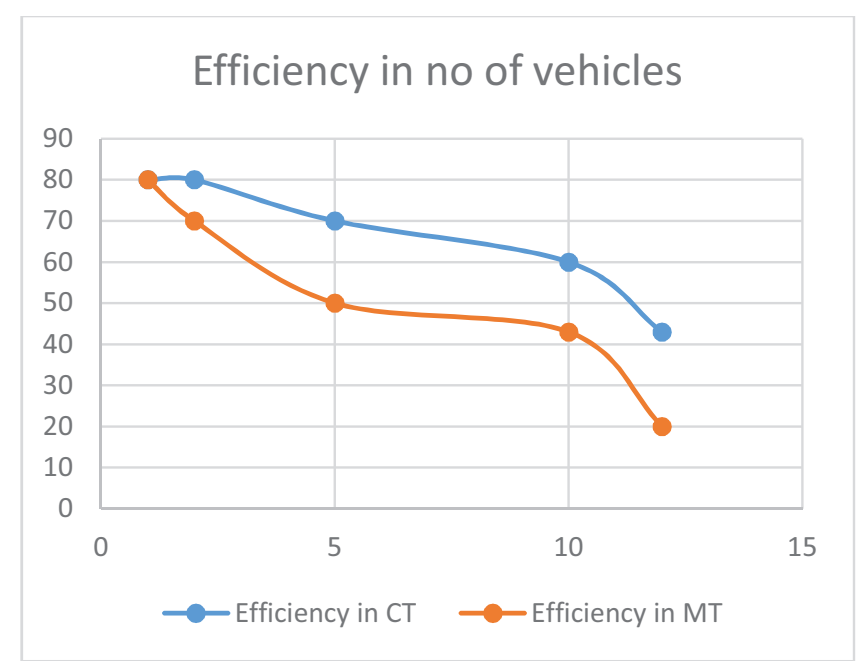

Figure 12: Efficiency of tracking of the proposed system on frame rate

\section{Efficiency in Frame rate}

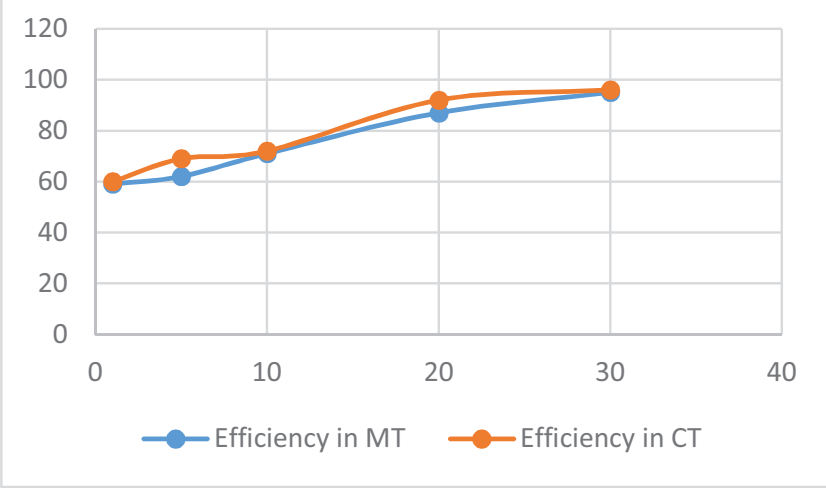

Figure 13: Efficiency of tracking of the proposed system on frame rate

TABLE IV EFFICIENCY IN FRAME RATE

\begin{tabular}{|c|c|c|}
\hline Frame Rate & Efficiency in MT & Efficiency in CT \\
\hline 30 & 95 & 96 \\
\hline 20 & 87 & 92 \\
\hline 10 & 71 & 72 \\
\hline 5 & 62 & 69 \\
\hline 1 & 59 & 60 \\
\hline
\end{tabular}

Here, 90 frames are considered per video and 20 such videos are used as dataset taken from motorways of Bangladesh. The algorithm is simulated (using MATLAB) in still video frames. The method is analyzed with two sets.

A denotes segmentation with vehicle detection with Morphological features (MT) and B denotes Vehicle detection using correlation transform (CT). Detection accuracy is computed in terms of number of vehicles on road given in figure 12 and frame rate given in figure 13. Two graphs suggests that the proposed technique is better than the other two.

\section{CONCLUSION}

This research has significant applications such as: access control in security sensitive areas, securities for important buildings, detection of military target areas, traffic observation in cities and highways, detection of anomalies behavior, traffic control management for recognize vehicles that commit traffic violation, such as occupying lanes reserved for public transport, breaking speed limits, crossing red light, entering restricted area without permission; and among many other applications. Our major concern of research was centered to these applications and the system was built and tested accordingly.

For detection and tracking this system is working well with a greater efficiency, although certain limitations exists. 
- Haar Cascade is not suitable for mobile and lower ranged processor oriented devices as it processes floats.

- High quality imaging - the quality of the captured image should be high; else noises may outcome in the output.

- The camera must be fit such that the captured image or video will not suffer much decent angle of skewing and rotation.

- Due to not having so many night view dataset, we could not test many night view videos for vehicle tracking as well as detection.

- There is a maximum limit of 100 tracked positions, i.e. a trail cannot be more than 100 samples.

- There is a maximum blob tracking count of 8192 .

\section{ACKNOWLEDGEMENT}

This work has been supported by the Swedish Research Council under grant 2014-4251.

\section{REFERENCES}

[1] P. Viola and M. Jones, "Rapid object detection using a boosted cascade of simple features," in Computer Vision and Pattern Recognition, 2001.

[2] C. P. Papageorgiou, M. Oren and T. Poggio, "A general framework for object detection," in International Conference on Computer Vision, 1998.

[3] R. Lienhart and J. Maydt, "An extended set of Haar-like features for rapid object detection," in International Conference on Image Processing, 2002.

[4] C. Messom and A. Barczak, "Fast and Efficient Rotated Haar-like Features Using Rotated Integral Images," in Australian Conference on Robotics and Automation, 2006.

[5] D. K. Panda, "Motion detection, object classification and tracking for visual surveillance application.," $\mathrm{PhD}$ thesis, Department of Electronics and Communiation Engineering National Institute of Technology Rourkela, Odisha, India, 2012.

[6] M. Everingham, L. V. Gool, C. K. I. Williams, J. Winn, and A. Zisserman, "The Pascal Visual Object Classes (VOC) Challenge," International Journal of Computer Vision, 88(2):303-338, 2010.

[7] J.-Y. Chang, T.-H. Chan, and H.-C. Fu, "Day-and-night video based face identification," in 2015 Seventh International Conference on Advanced Computational Intelligence (ICACI), 2015.

[8] J. Masek, R. Burget, J. Karasek, V. Uher, and S. Guney, "Evolutionary improved object detector for ultrasound images," in 36th International Conference on Telecommunications and Signal Processing (TSP), 2013.

[9] Z. Chen, T. Ellis, and S. A. Velastin, "Vehicle detection, tracking and classification in urban traffic," in 15th International IEEE Conference on Intelligent
Transportation Systems (ITSC), 2012.

[10] X. Wang, L. Xu, H. Sun, J. Xin, and N. Zheng, "OnRoad Vehicle Detection and Tracking Using MMW Radar and Monovision Fusion," in IEEE Transactions on Intelligent Transportation Systems, 17(7):2075-2084, 2016.

[11] H. David and Athira T.A., "Improving the Performance of Vehicle Detection and Verification by Log Gabor Filter Optimization," in 2014 Fourth International Conference on Advances in Computing and Communications (ICACC), 2014.

[12] S. Shujuan, X. Zhize, W. Xingang, H. Guan, W. Wenqi and $\mathrm{X}$. De, "Real-time vehicle detection using HaarSURF mixed features and gentle AdaBoost classifier," in 27th Chinese Control and Decision Conference (CCDC), 2015.

[13] X. Zhuang, W. Kang and Q. Wu, "Real-time vehicle detection with foreground-based cascade classifier," in IET Image Processing 10(4):289-296, 2016.

[14] A. Broggi, E. Cardarelli, S. Cattani, P. Medici and M. Sabbatelli, "Vehicle detection for autonomous parking using a Soft-Cascade AdaBoost classifier," in IEEE Intelligent Vehicles Symposium Proceedings, 2014

[15] R. K. Satzoda and M. M. Trivedi, "Multi-part Vehicle Detection using Symmetry Derived Analysis and Active Learning," IEEE Transactions on Intelligent Transportation Systems, 17(4): 926 - 937, 2016.

[16] D. J. Fleet and Y. Weiss, "Optical Flow Estimation," in Paragios; et al. Handbook of Mathematical Models in Computer Vision. Springer. ISBN 0-387-26371-3, 2006.

[17] L. Zhang, X. Zhang, and Y. Liu, "Research of the Realtime Detection of Traffic Flow Based on OpenCV," in 2008 International Conference on Computer Science and Software Engineering, 2008.

[18] H. Nguyen, P. Tran-Huu, H. Tran, and S. Ha, "Improved Optical Flow Estimation In Traffic Monitoring System," in 2013 Third World Congress on Information and Communication Technologies (WICT), 2013.

[19] P. K. Bhaskar, S. Yong, and L. T. Jung, "Enhanced and Effective Parallel Optical Flow Method for Vehicle Detection and Tracking," in 2015 International Symposium on Mathematical Sciences and Computing Research (iSMSC), 2015. 